\title{
Images - Rare diagnosis of high-grade, undifferentiated pelvic sarcoma for urinary dysfunction in a young man
}

\author{
Jaehoon Kim ${ }^{1}$; Alaya Yassein ${ }^{1}$; Udi Blankstein ${ }^{1}$; Michael Bonert ${ }^{2}$; Anil Kapoor ${ }^{1}$ \\ ${ }^{1}$ McMaster Institute of Urology, Hamilton, ON, Canada; ${ }^{2}$ Department of Pathology and Molecular Medicine, \\ McMaster University, St. Joseph's Healthcare Hamilton, Hamilton, ON, Canada
}

Cite as: Kim J, Yassein A, Blankstein U, et al. Images - Rare diagnosis of high-grade, undifferentiated pelvic sarcoma for urinary dysfunction in a young man. Can Urol Assoc J 2020 August 7; Epub ahead of print. http://dx.doi.org/10.5489/cuaj.6703

Published online August 7, 2020

$* * *$

\section{Introduction}

Sarcomas are mesenchymal malignancies which represent approximately $1 \%$ of adult cancers. ${ }^{1}$ Pelvic sarcomas in adults are relatively rare and represent less than $5 \%$ of all adult sarcomas, the majority of which originate in the genitourinary tract. ${ }^{2}$ The initial presentation of pelvic masses tends to occur at an advanced stage with lower urinary tract symptoms, hematuria and voiding dysfunction. ${ }^{3}$ Multiple structure involvement may lead to challenges in diagnostic classification and treatment, but most pelvic sarcomas are classified as one of the following: leiomyosarcoma, rhabdomyosarcoma, or gastrointestinal stromal tumours. ${ }^{2,3}$ We present a case of high grade undifferentiated sarcoma in a young patient that was radiologically favoured to originate from the prostate.

\section{Case report}

A 33-year-old man presented to urology with a two-month history of abdominal and perineal pain, constipation, and voiding dysfunction (dysuria, frequency, urgency, poor stream). The patient received initial antibiotic treatment for presumed prostatitis, and was referred to urology when the therapy was unresponsive. He had no previous past medical or surgical history, was a lifetime non-smoker, and had no prior radiation exposure. In the clinic, digital rectal examination demonstrated a large prostate mass and bladder ultrasound demonstrated significant urinary retention $(>1 \mathrm{~L})$, which necessitated relief via Foley catheterization. The following investigations were all within normal limits: prostate specific antigen (PSA $0.88 \mathrm{ng} / \mathrm{mL}$ ), creatinine, urine microscopy, culture, and urine cytology. Flexible cystoscopy revealed a large mass protruding from the bladder trigone. Urgent computed tomography of the pelvis showed a $12.8 \times 10.2 \times 16.8$ $\mathrm{cm}$ necrotic mass appearing to arise from the prostate, multiple pathologically enlarged lymph nodes, and a $2.7 \mathrm{~cm}$ osteolytic lesion in left iliac bone. (Fig. 1) No other lesions suspicious for 
metastases were identified. A transrectal ultrasound-guided (TRUS) biopsy revealed high grade (FNCLCC grade 2 of 3) undifferentiated (spindle cell) sarcoma. (Fig. 2) Pathologic ancillary tests revealed markers that were diffusely positive (CD99), focally positive (CK18, CD56, S100, MyoD1, myogenin) and negative (desmin, caldesmon, smooth muscle actin, CD3, CD20, CD34, CD45, CD117, TdT, AE1/AE3, CAM5.2, EMA, estrogen receptor, progersterone receptor, WT1, Neuron-specific enolase, synaptophysin, chromogranin A). FISH (Vysis https://www.molecular.abbott) was negative for an EWSR1 gene rearrangement. TruSight FNA Fusion Panel (https://illumina.com) showed no actionable fusion genes. A subsequent biopsy of the iliac bone showed a similar appearing tumour in keeping with a metastasis.

The case was presented at multidisciplinary sarcoma rounds and the tumour was viewed as unresectable. After admission to the hospital with a pain crisis, the mass effect of the tumour had progressed to large bowel obstruction and bilateral hydronephrosis, which was managed with diverting colostomy and bilateral nephrostomy tubes. He also developed bilateral foot drop with grade 0 ankle dorsiflexion and eversion, presumed to be iliac lymph nodes impinging on the lumbosacral plexus. Neurology recommended conservative management with ankle-foot orthosis and physiotherapy. Inpatient palliative care was actively involved throughout admission to optimize pain control with opioids.

Due to worsening symptoms, the patient was transferred to the regional cancer centre to facilitate inpatient radiotherapy. Eight weeks after diagnosis, re-imaging showed significant increases in size of the primary tumour ( $14.4 \times 18.6 \times 29.5 \mathrm{~cm})$, lymph nodes, and osteolytic lesion; additionally, new sub-centimeter lung lesions and para-aortic lymph nodes were identified. (Fig. 3) In total, he received 10 fractions of radiotherapy, followed by chemotherapy ( 6 cycles capecitabine, 1 cycle doxorubicin). After developing sepsis, chemotherapy was discontinued and comfort measures were undertaken at a hospice facility. Unfortunately, he passed away within six months of his initial presentation.

\section{Discussion}

This unique case features a rapidly progressing malignancy presenting as voiding dysfunction in a young man. The biopsy of the tumour yielded a rare undifferentiated sarcoma, but the complete diagnosis remains unknown as there was no further resection or autopsy. The result of the biopsy could have been due to (1) a primary, de novo undifferentiated sarcoma or (2) a failure to extract more differentiated portions of this large tumour. Based on the clinical course of the case, further investigation (re-biopsy, resection) was not warranted for the patient.

Metastatic disease at presentation is associated with poor prognosis for urological sarcomas, and osteoclastic bone lesions have previously been associated with prostate sarcomas. ${ }^{4-6}$ En bloc resection with cytoreductive cystoprostatectomy was considered during early management, but judged to be too morbid and there has been no reports of long-term remission of any localized treatment (radiotherapy, cytoreductive surgery) for metastatic primary prostate sarcoma. ${ }^{3,4}$ In the literature, only a single case of oligometastatic disease showed 
improved survival after a metastatectomy ( $10 \mathrm{vs} 23$ months). ${ }^{7}$ Nodal disease occurs in $46 \%$ of sarcoma cases,${ }^{6}$ and our patient demonstrated enlarging iliac nodes that likely resulted in lumbosacral plexopathy. Pelvic sarcomas are extremely rare, thus there is limited evidence to conclusively determine the best management plan. Urinary and bowel obstruction from pelvic sarcomas, as seen in this case, have previously been documented. ${ }^{7}$

\section{Conclusions}

To our knowledge, this is the youngest case of a pelvic sarcoma presenting as voiding dysfunction in the literature. One previous case of a 46 year old with metastatic retroperitoneal spindle cell sarcoma presented with voiding dysfunction and underwent surgical resection with positive margins; his survival status was not disclosed. ${ }^{8}$ This case report highlights the aggressiveness of an undifferentiated spindle cell sarcoma in the pelvis without any curative interventions. The clinical features of pelvic sarcomas are often unremarkable until significant tumour growth has already occurred, and is often overlooked as a diagnosis in young men. In such patients who are unresponsive to initial treatment for recurrent prostatitis, further workup may reveal such rare diagnoses. 


\section{Images: High-grade, undifferentiated sarcoma}

\section{References}

1. Burningham Z, Hashibe M, Spector L, et al. The Epidemiology of Sarcoma. Clin Sarcoma Res; 2: 14.

2. Keyzer-Dekker CMG, Houtkamp RG, Peterse JL, et al. Adult Pelvic Sarcomas: A Heterogeneous Collection of Sarcomas? Sarcoma; 8: 19.

3. Musser JE, Assel M, Mashni JW, et al. Adult prostate sarcoma: the Memorial Sloan Kettering experience. Urology; 84: 624-8.

4. Lee G, Lee SY, Seo S, et al. Prognostic factors and clinical outcomes of urological soft tissue sarcomas. Korean J Urol; 52: 669-73.

5. Dotan ZA, Tal R, Golijanin D, et al. Adult Genitourinary Sarcoma: The 25-Year Memorial Sloan-Kettering Experience. J Urol; 176: 2033-2039.

6. Andreou A, Whitten C, MacVicar D, et al. Imaging appearance of sarcomas of the prostate. Cancer Imaging; 13: 228-37.

7. Wang X, Liu L, Tang H, et al. Twenty-five Cases of Adult Prostate Sarcoma Treated at a High-volume Institution From 1989 to 2009. Urology; 82: 160-165.

8. Agafonoff S, Vaidya SK, DeFade B. High-grade undifferentiated pleomorphic sarcoma of the pelvis: A case report and review of literature. Urol Ann; 8: 372.

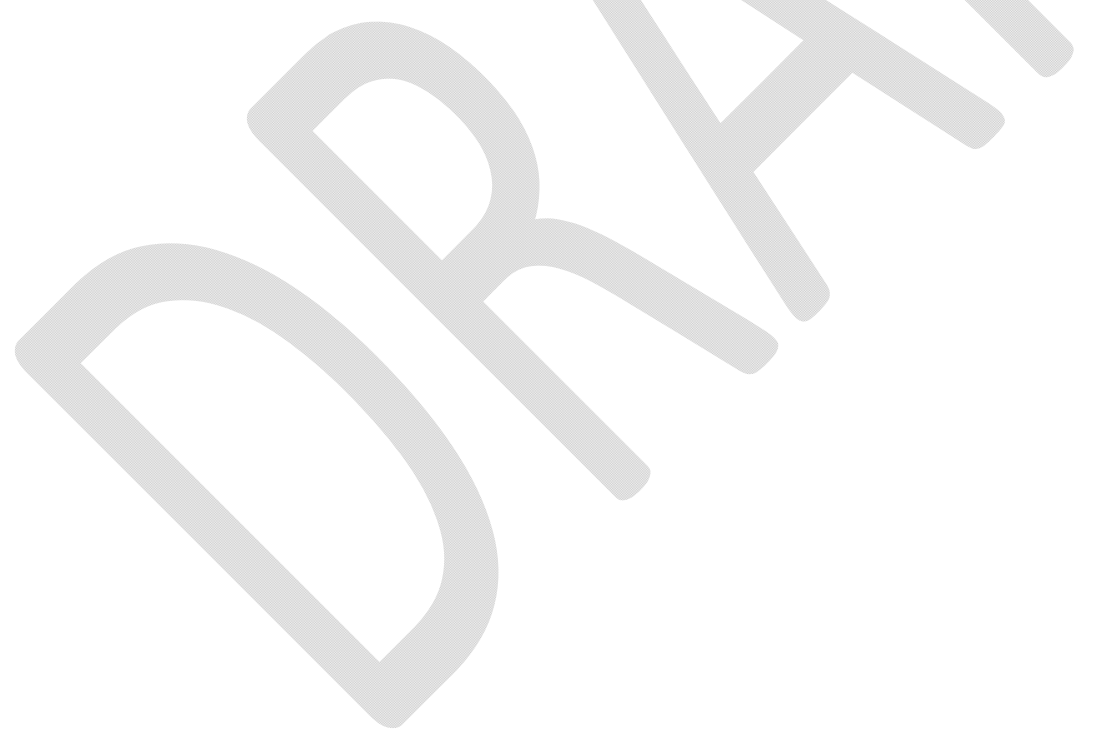




\section{Figures and Tables}

Fig. 1. Computed tomography of the abdomen and pelvis at initial presentation, demonstrating 12.8 x 10.2 x $16.8 \mathrm{~cm}$ mass. From left to right, coronal view, axial view, and sagittal view. B: bladder; P: prostate.
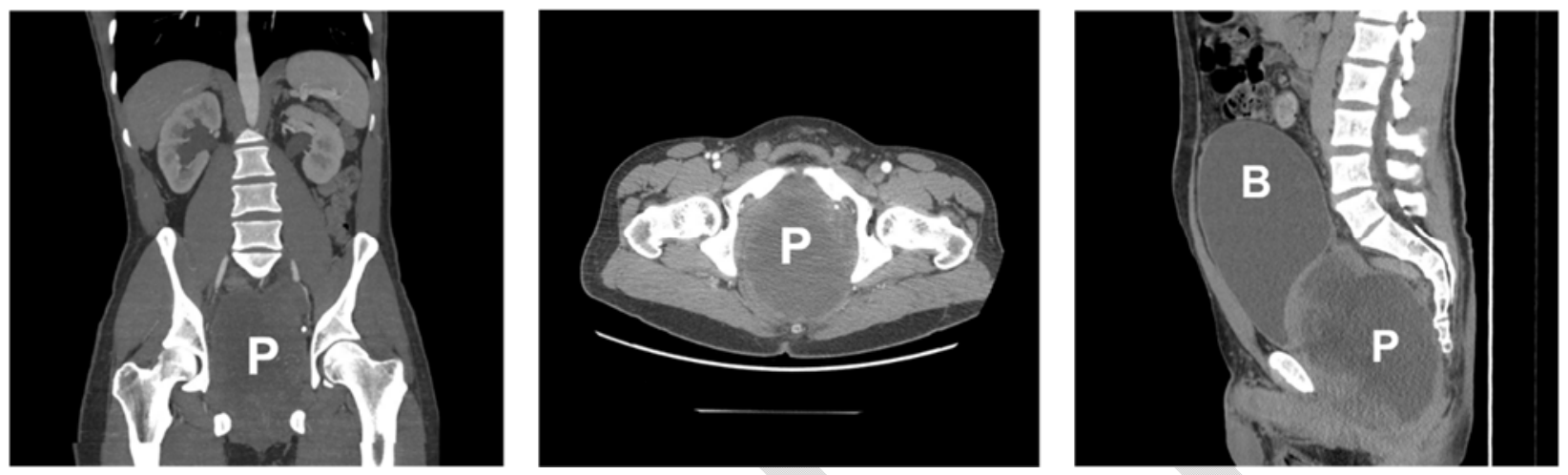

Fig. 2. Photomicrographs of the cellular undifferentiated prostatic spindle cell tumour (top-left) and histomorphologically similar iliac bone tumor (top-right), both haematoxylin and eosin stain; positive CD99 immunostain in the prostatic tumour (bottom-left); negative CD34 immunostain in the prostatic tumour (bottom-right). The focal CD34 positive staining is a blood vessel. Each top and each bottom image shows an area of $0.84 \mathrm{~mm} \times 0.56 \mathrm{~mm}$ and $0.42 \mathrm{~mm} \times 0.28 \mathrm{~mm}$, respectively.
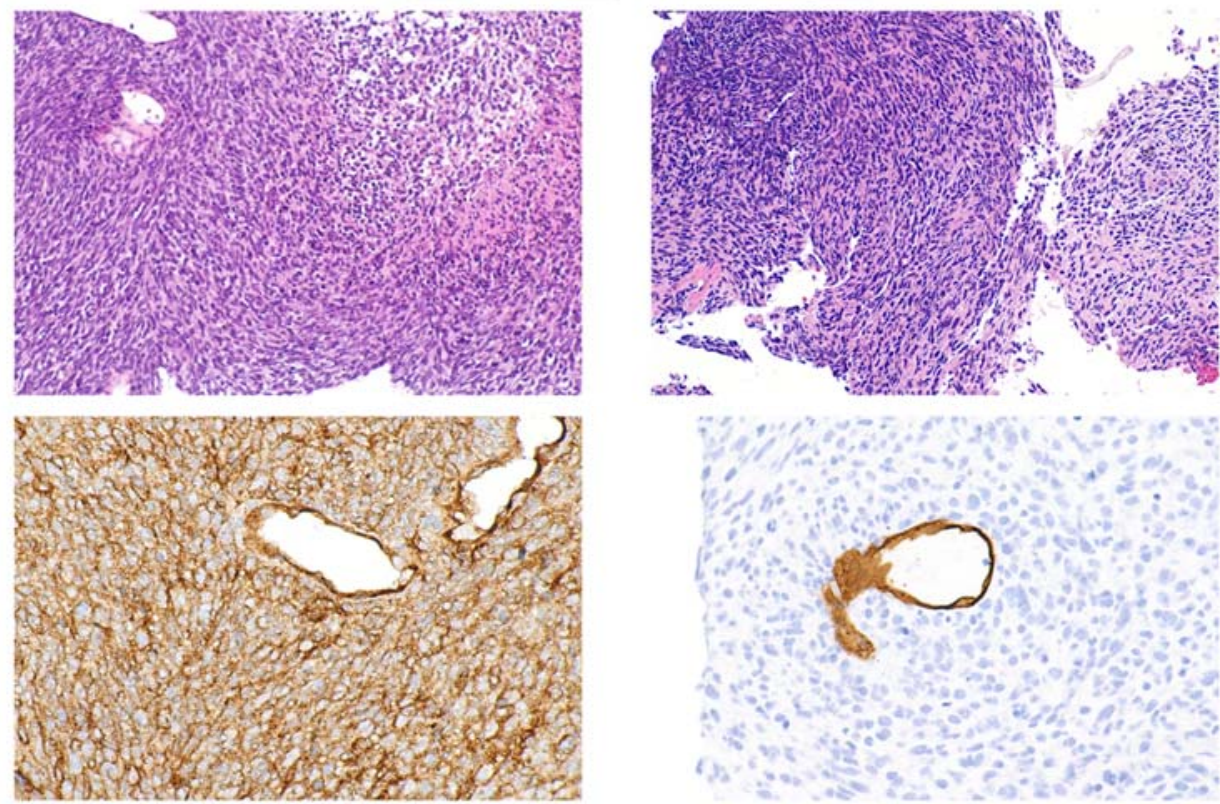
Fig. 3. Sagittal view of computed tomography of the abdomen and pelvis done at eight weeks after initial presentation, demonstrating a 14.4 x 18.6 × $29.5 \mathrm{~cm}$ mass. B: bladder; P: prostate.

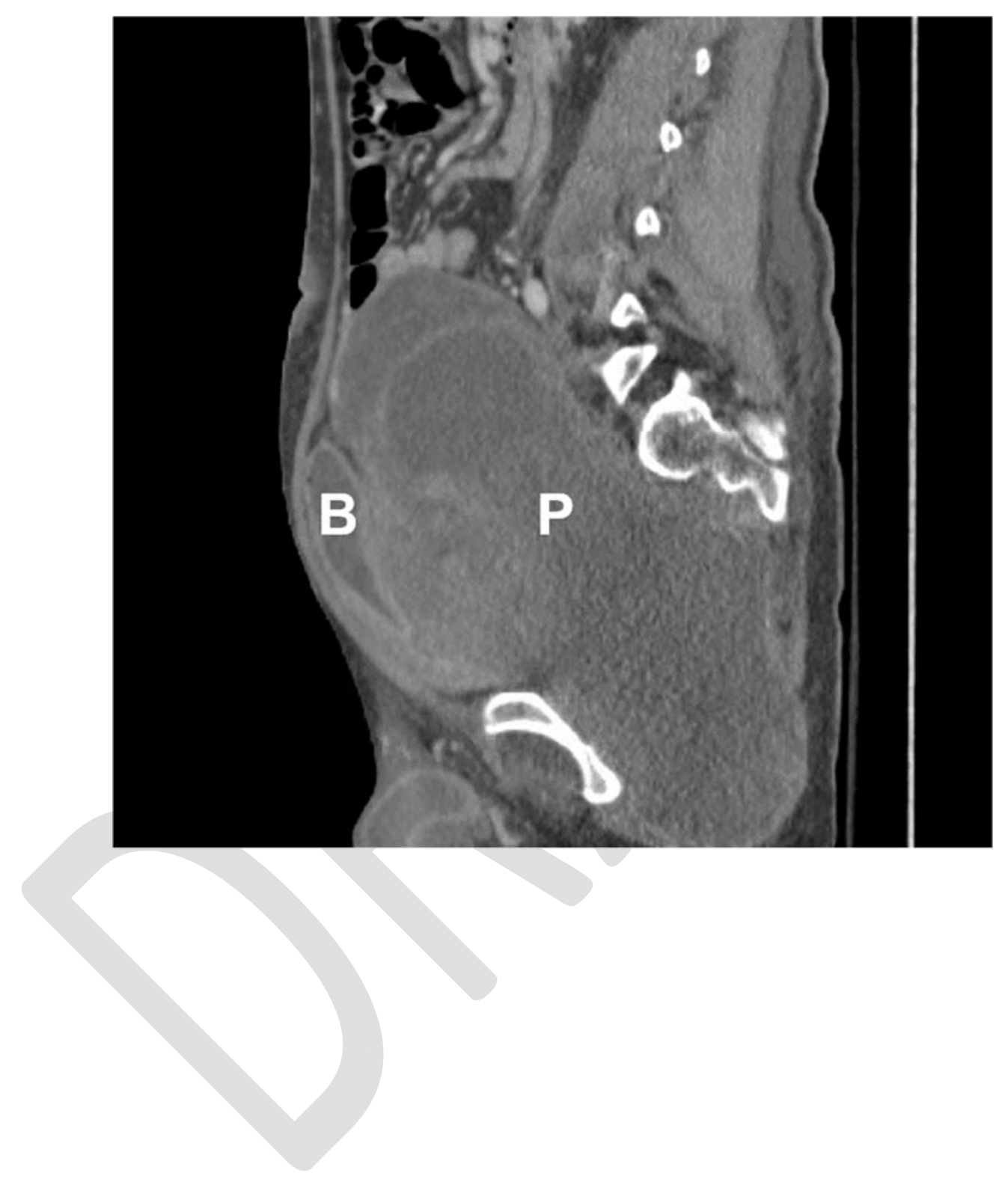

